\title{
The Status of Interventional Radiology as a Specialty among Medical Students in India-Knowledge, Interest, and Exposure
}

\author{
Bibin Sebastian ${ }^{1, \odot ~ S h y a m k u m a r ~ N . ~ K e s h a v a ~}{ }^{2, \odot}$ Raghuram Lakshminarayan ${ }^{1, \odot}$ \\ ${ }^{1}$ Division of Vascular Radiology, Department of Radiology, Hull \\ University Teaching Hospitals NHS Trust, Hull, United Kingdom \\ ${ }^{2}$ Department of Interventional Radiology, Christian Medical College, \\ Vellore, India

\begin{abstract}
Address for correspondence Bibin Sebastian, MBBS, MD, DNB, EDIR, FRCR, Division of Vascular Radiology, Department of Radiology, Hull University Teaching Hospitals NHS Trust, Hull HU3 2JZ, United Kingdom (e-mail: drbibinsebastian@gmail.com).
\end{abstract}

\begin{abstract}
Keywords

$-I R$

- IR curriculum

- medical education

- medical students

- online survey

Purpose Interventional radiology (IR) is a young medical specialty where image guidance is utilized in minimally invasive procedures as a treatment option and/or as a diagnostic tool. There is an exponential increase in awareness and interest in $I R$ amongst medical students. This trend is continuing despite lack of proportionate representation of IR in the current medical curriculum. This study aims to understand the exposure to IR as a specialty amongst medical students in India.

Materials and Methods Anonymous, voluntary, online questionnaire was sent to medical students from different parts of the country. The survey comprised 15 questions regarding exposure and awareness on IR.

Results The responses were obtained from 1,024 medical students from 98 medical colleges across the country, majority (57.0\%) in the clinical years of their training. Thirty-six percent of them were interested in an IR career. Lack of awareness was the most (61.6\%) cited reason for not choosing an IR career. Majority (57.9\%) would consider IR as a clinical management option and believe that IR holds an important place in medical practice (68.4\%).

Conclusion There is an evident under-representation of IR in the medical curriculum. However, an increasing awareness and interest among medical students toward IR as a specialty is demonstrated. Incorporation of IR into current medical curriculum in a systematic way is the need of the hour. This would ultimately benefit a wide cohort of patients across multiple specialties.
\end{abstract}

\section{Introduction}

Interventional radiology (IR) is gaining wide acceptance as an independent specialty in modern medicine. Since the first angioplasty on January $16^{\text {th }}, 1964$ by Charles Dotter, IR has shown tremendous growth and has become an integral part

published online July 28,2021
DOI https://doi.org/

$10.1055 / \mathrm{s}-0041-1733813$ ISSN 0971-3026 of the patient management, sometimes the only life-saving option. There are independent IR residency programs currently being approved in various parts of the world. For instance, in United States the integrated IR and diagnostic radiology (DR) residency received Accreditation Council for Graduate Medical Education approval in 2014. In the United
(C) 2021. Indian Radiological Association.

This is an open access article published by Thieme under the terms of the Creative Commons Attribution-NonDerivative-NonCommercial-License, permitting copying and reproduction so long as the original work is given appropriate credit. Contents may not be used for commercial purposes, or adapted, remixed, transformed or built upon. (https://creativecommons.org/licenses/by-nc-nd/4.0/). Thieme Medical and Scientific Publishers Private Ltd. A-12, Second Floor, Sector -2, NOIDA -201301, India 
Kingdom IR gained subspecialty status in 2010. Within a short span of its introduction, IR has become one of the most sought after specialties. In India, there are several IR fellowship programs of 1 to 2 years duration after the radiology residency. In addition, 3-year super-specialty training courses have been introduced; these are DM (Doctor of Medicine) by Medical Council of India and DNB (Diplomate of National board) by National Board of India. ${ }^{1,2}$

DR is already integrated into the current medical curriculum right from the preclinical years of training. This is evident in the way anatomy and clinical subjects are taught. ${ }^{3,4}$ However, there is lack of representation for IR, both theoretical and clinical exposure. Most of the standard medical text books mentions IR treatment options. However, it is not dealt in detail within the scope of undergraduate training. Numerous studies have established this fact and further pointed out that this might discourage medical graduates in future, not only in choosing IR as a career but also in considering it as an alternative management option within their chosen subspecialty. ${ }^{5-9}$ Also, many studies have underlined the positive outcomes of introduction of an undergraduate IR curriculum. ${ }^{10,11}$

Expansion of IR services in concordance with the rapidly evolving health care system in India is currently nonexistent. Lack of awareness among other specialty colleagues, poor interest, and sparse formal IR training opportunities for aspiring medical graduates are amongst the various reasons for this. The root cause eventually lies in the current medical curriculum where IR has been under-represented. Rectifying this can solve a few of the challenges faced by interventional radiologists like shortage of personnel and inter-specialty conflicts. ${ }^{11}$ This study aims to ascertain the current status of IR in medical students by assessing the knowledge, exposure, and interest of IR among students from medical colleges in various parts of the country.

\section{Materials and Methods}

An anonymous, voluntary, online questionnaire was completed by medical students pan-India between April 27 ${ }^{\text {th }}$, 2020 and May 06 ${ }^{\text {th }}, 2020$. The local students' community representatives were contacted and requested to volunteer in disseminating the information about the survey. The students were informed about the survey and the online link to questionnaire was posted in their common WhatsApp group. Responses were received from 1,024 medical students of 98 medical colleges. The questionnaire consisted of 15 mandatory questions including both multiple-choice questions and Yes/No responses, intended to assess the awareness and knowledge on IR, familiarity with IR procedures, exposure to IR, and interest in IR as a career ( - Fig. 1). The survey questions were formulated based on similar studies conducted in the past. ${ }^{9-12}$ However, questions 7 and 8 were newly introduced to know their specialty inclination and to assess the exposure to cardiology (one of the popular higher subspecialty). Survey responses were arranged on a spreadsheet, statistically analyzed and results were obtained.

\section{Results}

The 1,024 respondents comprised 529 (51.6\%) males and 495 (48.4\%) females. The majority (57\%) of the respondents were currently in the clinical years of their training (-Fig. 2). With regard to their inclination of a career choice, $28.4 \%$ chose medical and $23.0 \%$ chose surgical as their choice of specialty. At the same time, $20.5 \%$ responded as they have an interest in both core specialties alike while $28.1 \%$ had not decided yet.

Most (58.7\%) of the students had exposure to the radiology department. Interventional Radiology services were present in $48 \%$ of the respondents' institutions. However, only $16 \%$ had a teaching session or talk related to IR ever. Thirty-one percent of students responded that they have come across someone who has undergone an IR treatment. To the question, whether they have visited a catheterization laboratory and observed a coronary angiogram, the majority (80\%) gave a negative response.

Regarding their interest in radiology as a career, $42 \%$ responded positively, while $29 \%$ were unsure. About $36 \%$ of the students responded that they would consider IR as a career while $31 \%$ were unsure of this. Ninety-four out of 236 (39.8\%) surgical specialty inclined students would choose IR as well as radiology as a career. Ninety-six (32.3\%) and 126 (43.3\%) of 291 medical specialty inclined students would choose IR and radiology as a career, respectively. Also, 38.6\% of males and $33.6 \%$ of females are interested in IR as a career. Specialty inclination did not show a statistically significant influence on choosing IR or radiology as a career ( $p$-value is 0.104 ). The most common reason for not choosing IR as a career is lack of awareness about the specialty (61.6\%), followed by the already made decision on another specialty before exposure to IR (27.3\%). Wanting a relaxed lifestyle was the reason for $13.6 \%$ ( $15 \%$ of males and $13 \%$ of females). There were also reasons like lack of patient interaction, threat from artificial intelligence, health reasons (familial tremors), and lack of formal training programs, which altogether comprised less than a percent (-Fig. 3). However, 36 (3.5\%) students have already made up their mind for IR as a career.

The majority (57.9\%) of the students responded that they would consider IR as a management option for their patients in the future, while $31.3 \%$ were neutral in their response, and $7.4 \%$ were less likely to consider. However, $3.4 \%$ of students would never consider IR as a management option (-Fig. 4). Majority of respondents believed that the scope of IR is promising-excellent (39.4\%) and good (29\%) (- Fig. 5).

Regarding the familiarity with IR procedures, only 94 (9.2\%) students were aware that all the given nine IR procedures are performed by IRs. Neurointerventions like stroke and aneurysm coiling (74.2\%), followed by angioplasty and stenting of visceral and peripheral blood vessels (67.6\%) were the most familiar IR procedures. This was followed by the treatment for biliary obstruction (49.8\%), aortic aneurysm repair $(49.7 \%)$, varicose vein ablation and biopsies and drainages (44.9\%). Treatment for benign prostatic hyperplasia was the least familiar IR procedure (28.4\%) followed by uterine fibroid embolization (33.1\%) (-Fig. 6). 
This survey aims to assess the awareness level among medical students about this relatively new branch of medicine. The survey is voluntary and anonymous. By taking the survey you are consenting to let us, the investigators, use the data compiled. The study intends to ascertain the exposure to interventional radiology in the current medical curriculum. You will be asked a series of 15 questions, most of which are multiple choice format or Yes/No. Thank you for your time.

1. Gender:

o Male o Female

2. Name of the medical school:

3. Year of training: $0102 \circ 3 \circ 4$

4. Have you had any exposure to radiology department? o Yes o No

5. Is there Interventional radiology in your hospital?

o Yes o No o May be

6. Among the following what all procedures/treatments do you think are performed by an interventional radiologist? (check the box if yes)

- Stroke thrombectomy and aneurysm coiling

- Angioplasty and stenting of blood vessels

- Treatment for biliary obstruction

- Aortic aneurysm repair

- Varicose vein ablation

- Biopsies and drainages

o Chemotherapy for hepatocellular carcinoma

o Treatment for uterine fibroids

- Treatment for benign prostatic hyperplasia

7. Have you ever visited a Cath lab or observed a coronary angiogram?

o Yes o No

8. What's your specialty inclination?

o Medical o Surgical o Both o Not decided
9. Do you consider radiology as a career? o Yes o No

10. Do you consider interventional radiology as a career?

o Yes o No

11. Why would you NOT choose interventional radiology as a career?

- Already decided on another specialty

- Do not know much about interventional radiology

- Want a relaxed lifestyle

- Radiation exposure

- Others, please specify

12. Have you ever come across a person who underwent any kind of interventional radiology procedure?

o Yes o No

13. Have you ever had an educational session or a talk on interventional radiology? o Yes o No

14. How much likely do you consider interventional radiology as a treatment/management option?

- 1 Not at all o 2 Less likely o 3 Not sure 04 Likely o 5 Very likely

15. What do you think about the scope of interventional radiology?

- 1 Not at all o $2 \mathrm{Bad}$ o 3 Moderate 04 Good o 5 Excellent

Fig. 1 Survey questionnaire.

"Year 1 Year 2 Year 3 "Year 4

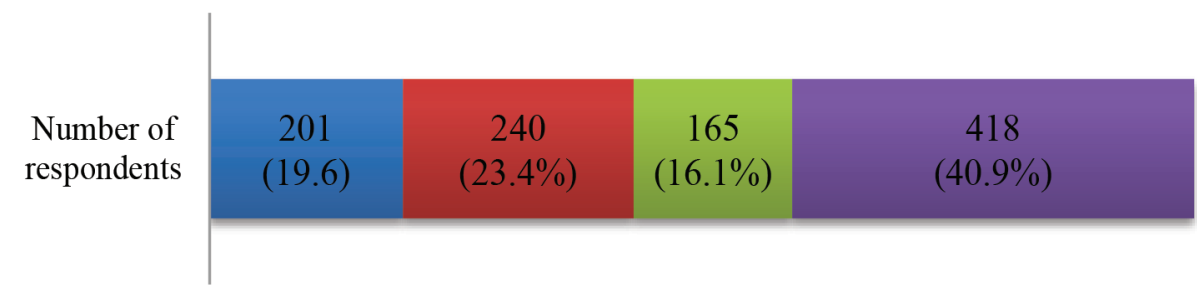

Fig. 2 Distribution of respondents based on the year of training. 


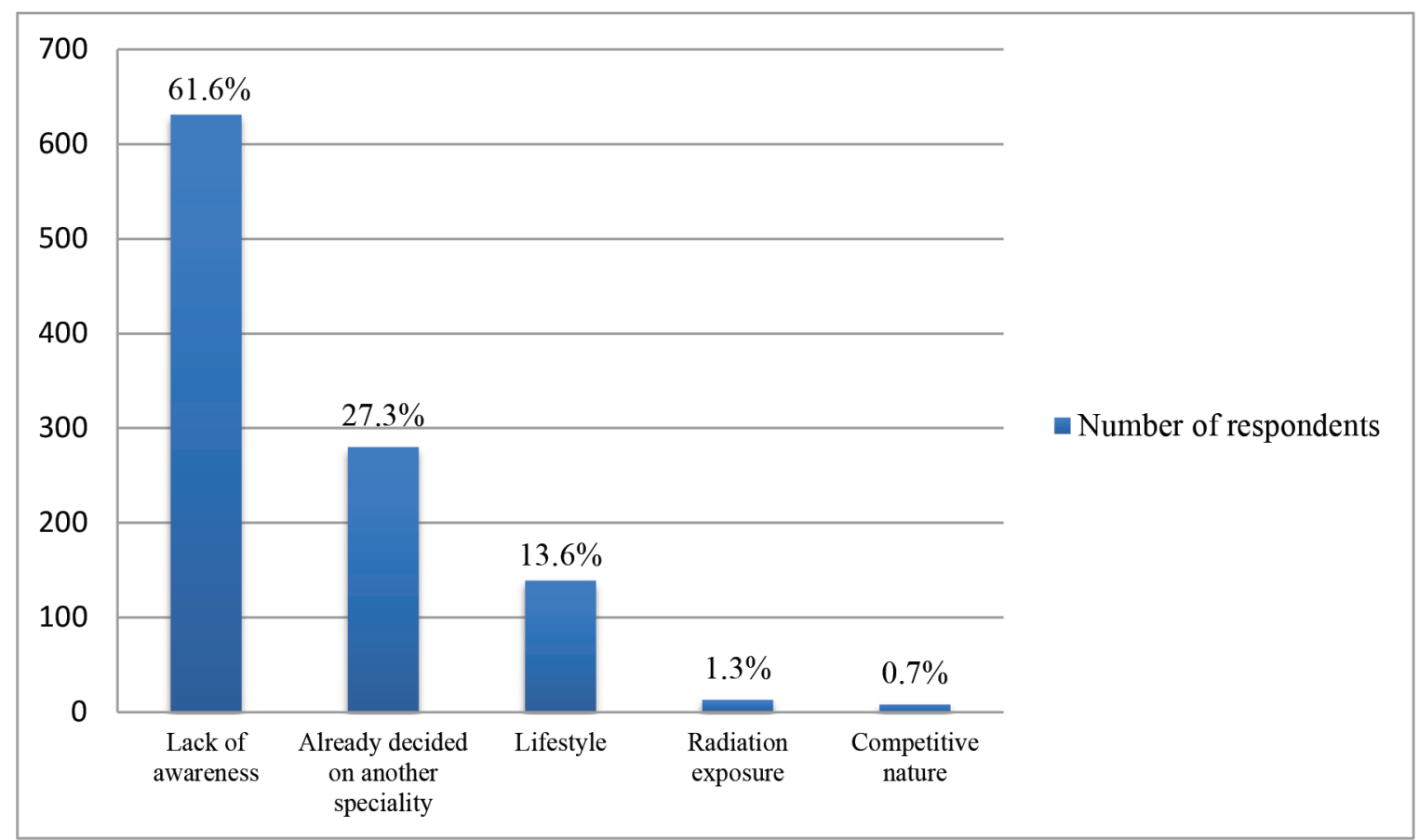

Fig. 3 Reasons for not considering IR as a career. IR, interventional radiology.

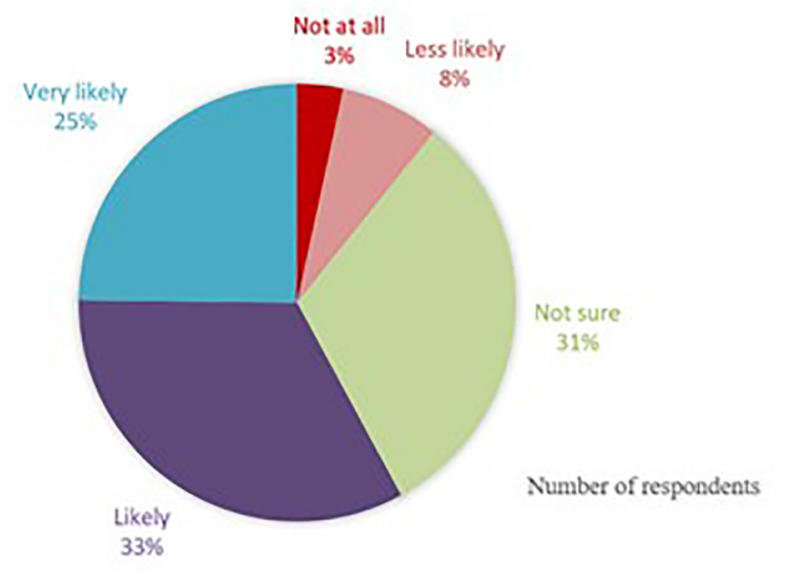

Fig. 4 The likelihood to consider IR as a management option. IR, interventional radiology.

\section{Discussion}

Recently, IR as a novel specialty has evoked a lot of interest among medical students and budding radiologists apart from colleagues practicing other specialties. To our knowledge, this is the largest such survey conducted among multiple institutions in a country involving the maximum number of medical students from all years. In this study $36 \%$ of respondents were interested in IR as a career. This varied from 15 to $73 \%$ in six previous studies in literature conducted at single institutions globally from 2009 to 2019.,3,7,7,10,12 There is a steady rising trend of interest for IR among medical students.

The majority of our participants were in their clinical years of medical training. Males choosing IR or radiology as their future career were more than females. Interestingly, the number of males citing lifestyle as a reason for not choosing IR was more than females. There was no statistically significant gender difference identified in our study regarding specialty inclination, interest in a career in IR or radiology, and lifestyle as a reason for not choosing IR.

Most of the students had exposure to radiology (58.7\%) and acknowledged that there are IR services provided in their institution (47.9\%). However, $40.3 \%$ of the students were not sure whether there was an IR provision. Similar to previous studies there is a statistically significant difference between the preclinical and clinical years medical students in their level of radiology exposure ( $p$-value $<0.01$ ) ${ }^{10,11}$ Also, $69.9 \%$ of the students responded that they have not come across anyone who had undergone an IR procedure. Not surprisingly, the exposure of medical students to other higher subspecialty procedures was also low. For example, cardiology is a popular higher subspecialty. However, approximately $80 \%$ of students responded that they have not visited a cardiac catheterization laboratory or observed an angiogram. Despite this, majority of students responded that they are interested in a career in IR (42\%) and 3.5\% of students have already decided to pursue an IR career. This could be due to the exposure to IR and its increasing awareness through various methods such as social media. Also, similar to a recent study by Foo et al, this indicates that there is increasing acceptance of IR among medical students as the years pass by. ${ }^{13}$

Regarding the familiarity of IR procedures, our study also had similar findings like the previous studies with angioplasty being recognized by many students as an IR procedure. ${ }^{11}$ However, the majority of students $(74.2 \%)$ opted for neurointerventional procedures like stroke thrombectomy and aneurysm coiling as procedures familiar to them as IR procedures. In a survey by O'Malley et al, among Canadian 


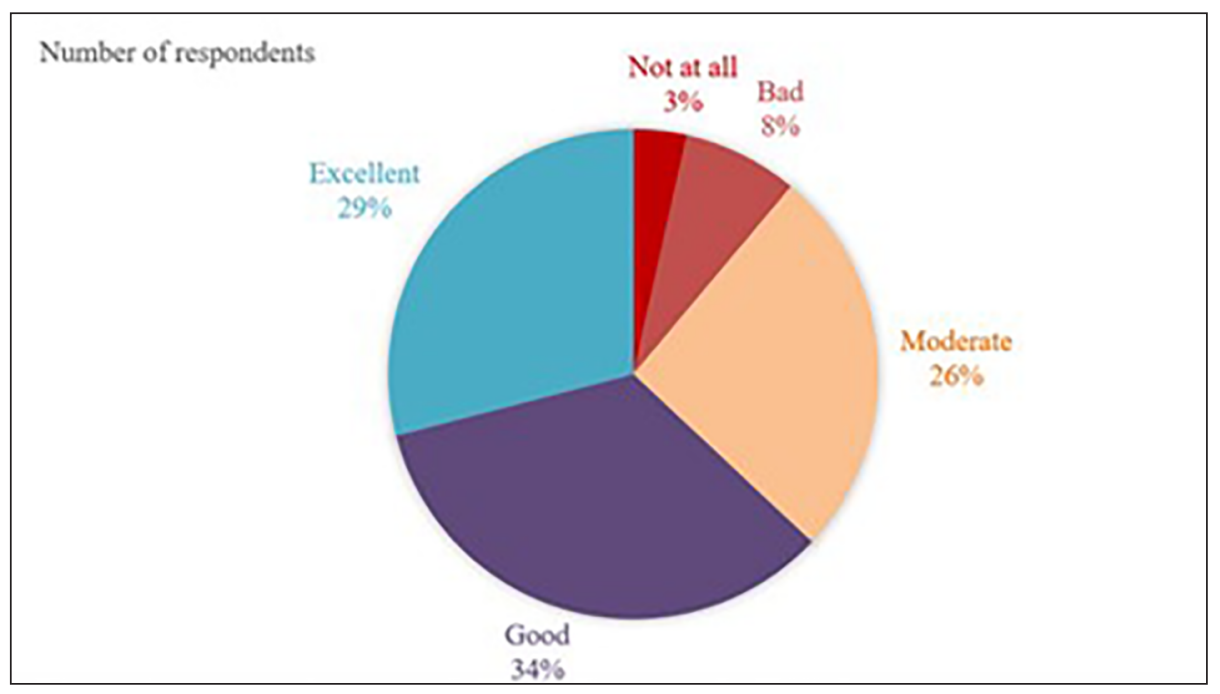

Fig. 5 Respondents' opinion about the scope of IR. IR, interventional radiology.

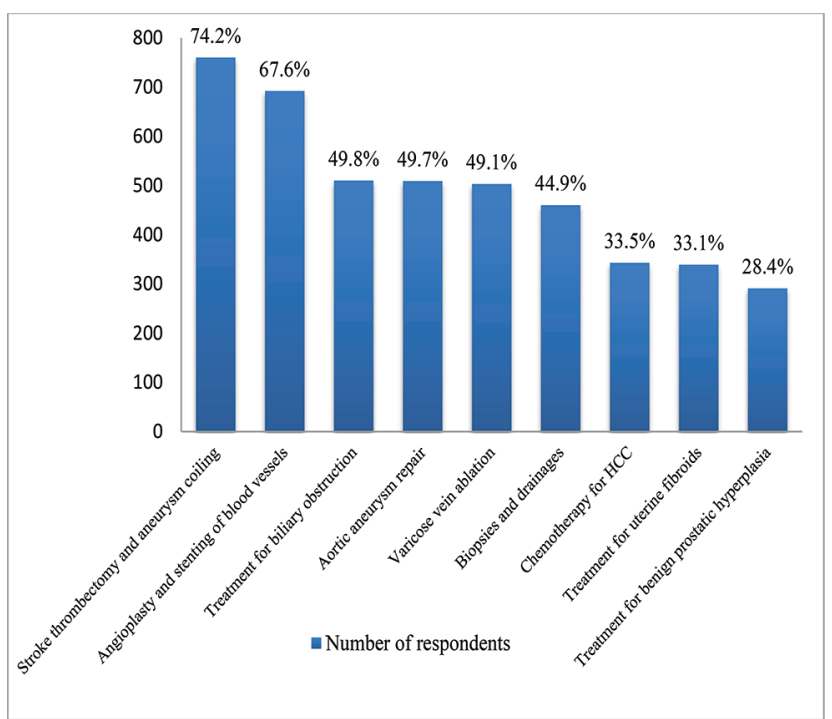

Fig. 6 Familiarity toward different IR procedures. IR, interventional radiology.

medical students in 2012 only 6\% of students responded that aneurysm coiling is performed by an interventional radiologist. ${ }^{9}$ We presume this may be due to the introduction of neuroradiology and interventions as a higher specialty in India and the increase in the number of neurointerventions performed by IRs. As expected, relatively newer procedures like prostatic artery embolization ("treatment for benign prostatic hyperplasia") was opted by the least number of respondents (28.4\%) as an IR procedure.

In our study, there was no statistically significant difference in the interest in IR as a career among students who had attended an IR teaching session/talk or have met someone who underwent an IR procedure or the presence of an IR department in their hospital versus those who did not have all these. We believe this may be due to the availability of information from various sources like social media, websites, and other specialty textbooks. This also highlights the fact that, if the medical students were provided with adequate exposure and knowledge about IR in a systematic method, the outcome could be beneficial. This has been identified by previous studies such as the one conducted by Shaikh et al among fourth-year Irish medical students in 2015. They found out that the knowledge $(6-45 \%)$ and interest in IR as a career $(60-73 \%)$ increased after the students attended a 10 -hour didactic lecture on IR. ${ }^{12}$

There is already a background interest amongst significant number of medical students on pursuing IR as a career. This could be due to the rapidly increasing acceptance and popularity of minimally invasive IR procedures among the medical professionals and the public, especially in the developed countries. We feel, as mentioned by some of the respondents, lack of formal training and the intense competitive nature of the current IR pathway may be one of the reasons holding medical students from contemplating IR as a career. Reduced number of IR procedures performed by IRs because of lack of referrals, either due to lack of awareness or due to \turf war" among physicians, also may be a reason.

This fuels the dire need for incorporation of an undergraduate IR curriculum in medical training in India. The Indian Society of Vascular and Interventional Radiology (ISVIR) and the Indian Radiological and Imaging Association (IRIA) have taken various steps toward this. This needs to be continued and taken forward with utmost importance. International professional organizations like the CIRSE, and the British Society of Interventional Radiologists have formulated IR curricula for medical students. ${ }^{14,15}$ These organizations have various programs to introduce medical students to the exciting world of IR. [S “Be InspIRed” is one such student program by the CIRSE where medical students are invited to attend their yearly congress for free to enjoy education and fun IR-related onsite sessions and events tailored especially to students' needs. ${ }^{16}$ Similarly, the ISVIR are also running programs during their annual conference including hands-on workshops on basic IR procedures, interactive sessions, and quiz programmes. ${ }^{17}$ 
Our study highlights the need to incorporate a systematic undergraduate IR medical curriculum in India. We need inclusion of IR procedure details in the standard textbooks as one of the management options wherever suited. Dedicated handbooks on IR for undergraduates could generate the interest for them to know more. Formal IR lectures by practicing expert IRs and IR rotations during third and final years may give some understanding about the IR specialty and can ignite interest amongst students. Designing a comprehensive undergraduate IR curriculum for India is essential. IR electives during internship have proved significantly effective in improving awareness and career interest in IR among medical students worldwide. ${ }^{18}$ One or 2 weeks electives dedicated to IR may be considered. Ultimately all these efforts would lead toward optimal patient care, for which after all we all strive for.

\section{Limitations}

This study has several limitations. The survey was conducted by sending access links to a Google Form through WhatsApp groups hence it was unable to assess the response rate. Even though, our survey population was comprised of students from multiple institutions all over the country there may be potential bias during this online survey. Nearly half of the study population was students from the preclinical years, who are relatively less exposed to, and less aware of IR as a specialty. Also, the study has not evaluated the potential methods of incorporating the undergraduate medical curriculum.

\section{Conclusion}

An online survey among medical students to assess their knowledge and attitude toward IR was feasible in a short period of time. Recently IR has generated a lot of interest like many other innovations in medicine among the medical fraternity and the public alike. Subsequently, the awareness and interest among medical students are also increasing, despite the lack of adequate representation of IR in the medical curriculum. There is an urgent need to incorporate a systematic undergraduate IR medical curriculum in developing countries like India. Medical students being the future IR specialists or the referring physicians deserve adequate exposure to this specialty and its role in the current practice of modern medicine. This enables future doctors to understand and practice modern medicine in a safe and effective manner, which in turn benefits many patients.

\section{Conflict of Interest}

None declared.

\section{References}

1 e-Gazette | NMC. Available at: https://www.nmc.org.in/e-gazette. Accessed June 13, 2021

2 Welcome To National Board Of Examination. Available at: https://natboard.edu.in/matter.php?notice_id=2012. Accessed June 13, 2021

3 DePietro DM, Kiefer RM, Redmond JW, et al. Increasing medical student exposure to IR through integration of IR into the gross anatomy course. J Vasc Interv Radiol 2017;28(10):1455-1460

4 Jafri NF, Wu P, Stanfield L, Slanetz PJ. Use of radiologic imaging to enhance physical diagnosis instruction in the preclinical curriculum. Acad Radiol 2008;15(7):942-947

5 Agrawal D, Renfrew MA, Singhal S, Bhansali Y. Awareness and knowledge of interventional radiology among medical students at an Indian institution. CVIR Endovasc 2019;2(1):45

6 Rajagopal R, Khera P, Garg P. Medical students and interventional radiology: miles to go. J Clin Interv Radiol 2018;02(3):206-206

7 Atiiga PA, Drozd M, Veettil R. Awareness, knowledge, and interest in interventional radiology among final year medical students in England. Clin Radiol 2017;72(9):795.e7-795.e12

8 Commander CW, Pabon-Ramos WM, Isaacson AJ, $\mathrm{Yu} \mathrm{H}$, Burke CT, Dixon RG. Assessing medical students' knowledge of IR at two American medical schools. J Vasc Interv Radiol 2014;25(11):1801-1806, 1807.e1-1807.e5

9 O'Malley L, Athreya S. Awareness and level of knowledge of interventional radiology among medical students at a Canadian institution. Acad Radiol 2012;19(7):894-901

10 Nissim L, Krupinski E, Hunter T, Taljanovic M. Exposure to, understanding of, and interest in interventional radiology in American medical students. Acad Radiol 2013;20(4):493-499

11 Leong S, Keeling AN, Lee MJ. A survey of interventional radiology awareness among final-year medical students in a European country. Cardiovasc Intervent Radiol 2009;32(4):623-629

12 Shaikh M, Shaygi B, Asadi $H$, et al. The Introduction of an Undergraduate Interventional Radiology (IR) Curriculum: impact on medical student knowledge and interest in IR. Cardiovasc Intervent Radiol 2016;39(4):514-521

13 Australian students' perspective on interventional radiology education: a prospective cross-institutional study-Foo2018-Journal of Medical Imaging and Radiation OncologyWiley Online Library. Available at: https://onlinelibrary.wiley. com/doi/full/10.1111/1754-9485.12764. Accessed May 14, 2020

14 IR curriculum for medical students. CIRSE. Available at: https://www.cirse.org/wp-content/uploads/2019/03/cirse IRcurriculum_medical_students_2019_web-1.pdf. Accessed May 14, 2020

15 Undergraduate IR. Curriculum | BSIR. Available at: https:// www.bsir.org/bsirt/ir-curriculum/undergraduate-ir-curriculum/. Accessed May 14, 2020

16 Student Programme-Be inspIRed... CIRSE. Available at: https://www.cirse.org/students/student-programme-be-inspired/. Accessed May 14, 2020

17 Keshava S, Palumbo A. Medical students and interventional radiology. J Clin Interv Radiol 2018;2(1):1

18 McGraw CJ, Loh CT, Ang MJ, et al. Impact of an interventional radiology elective on medical students. J Vasc Interv Radiol 2013;24(4):S69 